\title{
On the Possibility of Determining the Distribution of a Substance Penetrating into a Dense Medium Using Gamma-Ray Detection and Ranging Approach
}

\author{
L.L. Gurdev ${ }^{a}, *$ T.N. Dreischuh ${ }^{a}$, D.V. Stoyanov ${ }^{a}$ And C.N. Protochristov ${ }^{b}$ \\ ${ }^{a}$ Institute of Electronics, Bulgarian Academy of Sciences \\ 72 Tzarigradsko Shosse Blvd., 1784 Sofia, Bulgaria \\ ${ }^{b}$ Institute of Nuclear Research and Nuclear Energy, Bulgarian Academy of Sciences \\ 72 Tzarigradsko Shosse Blvd., 1784 Sofia, Bulgaria
}

(Received May 11, 2010)

\begin{abstract}
The possibility has been investigated of determining, using graydar (Gamma RAY Detection And Ranging) approach, the in-depth partial-density profile of some substance absorbed in a dense medium. Analytical algorithms have been derived for retrieving the in-depth profile of the partial density of the absorbed substance on the basis of the conjecturally known in-depth profile of the extinction of the absorbing medium and the experimentally determinable graydar profile. The retrieval error under the Poisson noise conditions has also been estimated analytically. The simulations performed of the Poisson-noise effect concern the case of soil moisture. The results obtained confirm the validity of the derived retrieval algorithms and error estimates and show that the soil moisture profile may be accurately determined to depths of $50 \mathrm{~cm}$, depending on the dry-soil bulk density, the sensing photon flux, and the measurement time.
\end{abstract}

PACS numbers: 07.85.-m, 81.70.-q, 92.40.Lg, 93.85.Np

\section{Introduction}

There are various single-sided and high-resolution gamma or X-ray techniques developed [1-9] for non-destructive evaluation of material substances that are not transparent for optical or microwave radiation. These methods are intended for determination of the electron-density distribution within the investigated objects. They are usually based on the dependence of the energy of the Compton single-scattered photons on the angle of scattering.

A common difficulty of them is the lack of any clear approach for taking into account the linear attenuation (extinction) within the object. Moreover, some of the methods $[3,4,6-9]$ require too complicated image-reconstruction algorithms like those in computer-aided tomography $[10-12]$. Other ones $[1,2,5]$ require relatively long data-collection time.

The graydar (Gamma RAY Detection And Ranging) approach [13-16] is free of the above-mentioned difficulties. Instead, it allows to determine simultaneously, in a relatively simple unambiguous one-sided way and with controllable accuracy and resolution, the profiles of the extinction and Compton-backscattering coefficients within an object of interest. This in turn would allow one to determine the internal distribution of differ-

* corresponding author; e-mail: lugurdev@ie.bas.bg ent constituent substances and their mass density. The capabilities of the graydar sensing have been studied and demonstrated in [13, 14], where three typical cases have been considered. In the first case, the graydar line of sight (LOS, sensing beam axis) penetrates homogeneous regions of different substances. In the second case, the probed object consists of only one material having nonuniform spatial density distribution. In the third case, the LOS penetrates homogeneous one-material ingredients surrounded by one-material medium with nonuniform density distribution. The procedure of laterally scanning the LOS and obtaining two-dimensional (2D) images (sections) of the probed objects has also been simulated. It is shown, for instance, that, at a number of $10^{9}$ sensing photons deposited along one LOS, one may establish with 2-3 mm longitudinal and transversal resolution the presence, the disposition, the shape, and the kind of different homogeneous ingredients, cavities and flaws within a homogeneous surrounding material (aluminum); the presence of more than one flaw along one LOS is shown to not lead to noticeable masking effect [15]. It is shown as well that the graydar approach is capable of finding, identifying, and imaging homogeneous ingredients (plastic TNT landmines) in homogeneous or inhomogeneous soil, at depths to $20 \mathrm{~cm}$, density-contrast from $5 \%-8 \%$ to $20 \%$, and longitudinal (along the LOS) and transversal resolution of $1-2 \mathrm{~mm}$ and $8-10 \mathrm{~mm}$, respectively $[14,16]$. 
The main purpose of the present work is to consider a somewhat different problem of graydar sensing compared to those mentioned above. This problem concerns in general the case when some known substance has been diffused into a dense medium with known gamma-ray extinction distribution. The task to be solved is to determine the LOS partial density profile of the diffused substance. An applied problem of this type is the determination of moisture distribution in soil. In the further analysis we shall investigate analytically the possibility of solving in principle the above-formulated general task. The results obtained will be in a sense tested and illustrated by simulating the Poisson noise influence on the measurement accuracy in the important for applications, concrete case concerning the soil moisture.

\section{Graydar principle and graydar equation}

Like the lidar (LIght Detection And Ranging) principle, the graydar principle consists in general in time-to-range resolved detection of the backscattering-due radiative return from a probed object irradiated by narrow-beam, pulsed gamma radiation. Considering an inelastic scattering process, the incident-photon energy may be denoted by $E_{\mathrm{f}}$, and the return-photon energy, by $E_{\mathrm{b}}$. During the detection procedure the radiative return is transformed into a return electrical signal $F(t)$ measured as a function of the time delay $t$ after the instant of emission of the corresponding sensing pulse. Under single-scattering conditions there exists one-to-one correspondence $t \equiv 2 z / c(z \equiv c t / 2)$ between the time $t$ and the LOS distance $z$ to the sensing-pulse front that is the front of the scattering volume contributing to the signal at this time; $c$ is the speed of light. Also, if $\Delta t$ is the sampling interval in the time domain, $\Delta z=c \Delta t / 2$ will be the sampling interval in the range domain. Thus, in practice, a time-to-range resolved return signal or graydar profile $F=F(t, \Delta t)=F(z, \Delta z)$ can be obtained. The main instrument for quantitative analysis concerning graydar is the so-called single-backscattering graydar equation [13] that describes the relation between the graydar profile $F(t, \Delta t)$, the parameters of the radiation-transceiving system (incident-photon rate, receiving detector area, receiving efficiency, sampling interval in the time or range domain, etc.), the energy $E_{\mathrm{f}}$ of the sensing gamma photons, and the LOS distribution of the physical characteristics (extinction and backscattering coefficients) of the medium under investigation. It is valid when only once backscattered and many times forward scattered photons are detected, and the pulse length $l_{\mathrm{p}}=c \tau_{\mathrm{p}}\left(\tau_{\mathrm{p}}\right.$ is the pulse duration) and $\Delta z$ are smaller than the least variation scale of the material characteristics inside the probed object. In this case the timing uncertainty $\Delta t_{\mathrm{u}}$ due to inertness and noise in the transceiving electronics should be much less than the larger of $\tau_{\mathrm{p}}$ and $\Delta t$. The achievable minimum range-resolution cell is then equal to $\max \left(l_{\mathrm{p}}, \Delta z\right)$.

When the scattering mechanism of concern is the Compton effect, two mutually complementary ways may be used of selecting the single-backscattering photons alone. The first way consists in ensuring as narrow as possible field of view of the radiation-receiving system. At the same time, the field of view should cover the sensing photon beam in order to avoid the loss of signal photons. Thus, the transversal beam size determines the minimum transversal-resolution cell. The second way consists in energy selection based on the dependence of the energy of the Compton-scattered photons on the scattering angle. So, according to the Compton formula [17], for $E_{\mathrm{f}}=511 \mathrm{keV}$ the signal (once only backscattered) photons will have Compton energy $E_{\mathrm{bc}} \approx 170 \mathrm{keV}$. The energy selection of the signal photons requires the use of photon detectors of determinate, sufficiently high energy resolution consistent with the Doppler broadening $[18,19]$, with half-width $\Delta E_{\mathrm{D}}$, of the signal-photon energy around $E_{\mathrm{bc}}$. It would be effective when sensing "light to medium-weight" materials causing relatively small Doppler broadening. In the case of "heavy" materials the broadening effect will be larger and the question about the disturbance of the energy selection requires a separate profound investigation.

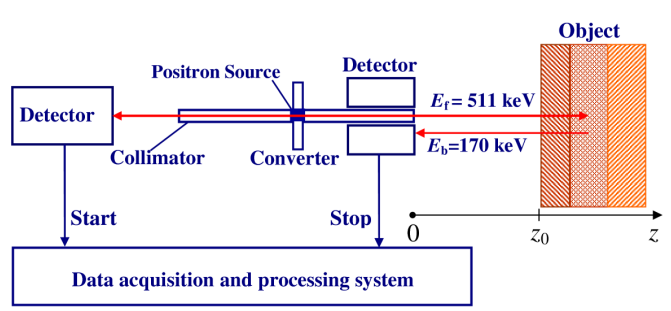

Fig. 1. Principle block-scheme of experimental arrangement for gamma-ray lidar-type in-depth sensing of optically opaque media.

A regime of $\delta$-pulse sensing could be achieved [13] by using a monostatic sensing system (see Fig. 1) emitting spontaneously a narrow beam of incident on the probed object gamma photons of energy $E_{\mathrm{f}}$ and detecting the Compton backward-scattered photons of energy $E_{\mathrm{bc}}$. The mean incident photon rate and the total measurement time may be denoted in this case by $q_{0}$ and $T$, respectively. Further, one may consider each incident photon as a $\delta$-like sensing pulse and specify the corresponding eventually registered signal photon by its energy $E_{\mathrm{b}}=E_{\mathrm{bc}}$ and arrival time $t$ with respect to the instant of emission of the sensing photon. To mark the instants of emission of the incident photons one may use $[13,20,21]$ sensing photon beams resulting from electron-positron annihilation within a converter irradiated by positrons from a radioactive source (Fig. 1). As a result of annihilation, two gamma photons of energy $E_{\mathrm{f}}=511 \mathrm{keV}$ are simultaneously emitted in opposite directions. A part of the annihilation photons, through a collimator, is formed as a sensing beam and directed to the probed object. The backpropagating photon of each "sensing" pair through the collimator reaches a scintillator and generates a start light pulse. The corresponding 
once only Compton backscattered signal photon of energy $E_{\mathrm{b}} \approx E_{\mathrm{bc}}=E_{\mathrm{f}} / 3$ reaches another scintillator and produces a stop light pulse. Both the light pulses produce in turn, through a photomultiplier, a couple of two time-shifted start and stop electronic pulses. These output pulses are further amplified and processed to identify simultaneously both the energy and the arrival time of each detected photon. During the measurement pe$\operatorname{riod} T$, the number of the sensing photons deposited into the probed object along one line of sight will be $q_{0} T$. At the same time, a realization $\widehat{N}_{T}\left(E_{\mathrm{bc}}, t, \Delta t\right)$ will be obtained of the energy-selected time-to-range resolved graydar profile $N_{T}\left(E_{\mathrm{bc}}, t, \Delta t\right)$ describing the mean number of accumulated signal gamma-photon counts per one resolution cell $\Delta t$, with arrival times between $t$ and $t+\Delta t$ corresponding to acts of backscattering between the points $z$ and $z+\Delta z$ along the LOS. The graydar profile is described by the equation [13]:

$$
\begin{aligned}
& N_{T}\left(E_{\mathrm{bc}}, t, \Delta t\right) \equiv N_{T}\left(E_{\mathrm{bc}}, z, \Delta z\right) \\
& \quad=d q_{0} \Delta z T z^{-2} \eta(z) \beta(z) \exp \left(-\int_{z_{0}}^{z} \mathrm{~d} z^{\prime} \alpha\left(z^{\prime}\right)\right),
\end{aligned}
$$

where $d=\pi\left(r_{\mathrm{d}}^{2}-r_{\mathrm{c}}^{2}\right)$ is the receiving detector area, $r_{\mathrm{d}}$ and $r_{\mathrm{c}}\left(\ll r_{\mathrm{d}}\right)$ are, respectively, the radii of the circular scintillating detector's layer and collimator's aperture, $z_{0}$ is the longitudinal coordinate of the "entrance" into the investigated object (see Fig. 1), $\eta(z)$ is the receiving efficiency of the experimental setup that is usually equal to unity [13], $\beta(z)\left[\mathrm{m}^{-1} / \mathrm{sr}\right]$ is the LOS profile of the volume backscattering coefficient, and $\alpha(z)=\alpha_{\mathrm{f}}(z)+\alpha_{\mathrm{b}}(z)$ is the profile of the two-way extinction index, $\alpha_{\mathrm{f}}(z)\left[\mathrm{m}^{-1}\right]$ and $\alpha_{\mathrm{b}}(z)\left[\mathrm{m}^{-1}\right]$ are the extinction coefficients for the incident and signal photons, respectively. Equation (1) is the $\delta$-pulse single-backscattering graydar equation.

For a one-material medium the variability of the profiles $\beta(z)$ and $\alpha(z)$ with $z$ is entirely defined, as a factor, by the corresponding (partial or bulk or natural-state) mass-density profile $\rho(z)$; besides, both the profiles are related through an independent of $z$, constant graydar ratio $b=\beta(z) / \alpha(z)$ [13]. For evaluating the coefficients $\beta$ and $\alpha$ of a great variety of monoatomic and polyatomic materials, the rich contemporary information may be used, given, e.g. in [22] and [23], about their natural density $\rho$, mass attenuation $\mu=\alpha / \rho$, and effective atomic number-to-mass ratio $\langle Z / A\rangle$.

\section{Determining the in-depth partial-density profile of a substance penetrating into a dense medium}

Consider the procedure of in-depth sensing of a dense two-component object consisting for instance of a "dry" porous component, e.g., dry soil, that has absorbed another, "liquid" component, e.g., water. The in-depth profiles of the volume backscattering coefficient $\beta_{\mathrm{ds}}(z)$ and the two-way extinction coefficient $\alpha_{\mathrm{ds}}(z)$ of the dry component will be supposed known. They can be preliminarily measured, also by graydar [13, 14], before absorbing the moist component. The problem to be solved consists in determining the partial-density profile $\rho_{\mathrm{w}}(z)$ of the liquid component on the basis of the measured graydar profile $N_{T}\left(E_{\mathrm{bc}}, t, \Delta t\right)$, the known experimental parameters $d, q_{0}, \Delta z, T$, and $\eta(z)$, and the known dry component characteristics $\beta_{\mathrm{ds}}(z)$ and $\alpha_{\mathrm{ds}}(z)$. For this purpose, Eq. (1) may be written in the form

$$
\begin{aligned}
& S(z)=\left[\beta_{\mathrm{ds}}(z)+\beta_{\mathrm{w}}(z)\right] \\
& \quad \times \exp \left(-\int_{z_{0}}^{z}\left[\alpha_{\mathrm{ds}}\left(z^{\prime}\right)+\alpha_{\mathrm{w}}\left(z^{\prime}\right)\right] \mathrm{d} z^{\prime}\right),
\end{aligned}
$$

where $S(z)=N_{T}\left(E_{\mathrm{bc}}, z, \Delta z\right) /\left[d q_{0} \Delta z T z^{-2} \eta(z)\right]$ is the so-called $S$-function, $\beta_{\mathrm{ds}}(z)+\beta_{\mathrm{w}}(z)=\beta(z), \alpha_{\mathrm{ds}}(z)+$ $\alpha_{\mathrm{w}}(z)=\alpha(z)$, and $\beta_{\mathrm{w}}(z)$ and $\alpha_{\mathrm{w}}(z)$ are the in-depth profiles of the volume backscattering coefficient and the two-way extinction coefficient of the liquid component.

Taking into account that $\beta_{\mathrm{w}}(z)=b_{\mathrm{w}} \alpha_{\mathrm{w}}(z)$ and $\alpha_{\mathrm{w}}(z)=\rho_{\mathrm{w}}(z) \mu_{\mathrm{w}}$, it follows that the problem of determining the profile $\rho_{\mathrm{w}}(z)$ consists in fact in solving Eq. (2) with respect to $\alpha_{\mathrm{w}}(z) ; b_{\mathrm{w}}$ and $\mu_{\mathrm{w}}=\mu_{\mathrm{wf}}+\mu_{\mathrm{wb}}$ are the graydar ratio and the mass attenuation index of the liquid component. The quantities $\mu_{\mathrm{wf}}$ and $\mu_{\mathrm{wb}}$ are the corresponding mass attenuation coefficients for the sensing photons and the backscattered photons. Note as well that, since the natural free-state water density is equal to $1 \mathrm{~g} / \mathrm{cm}^{3}$, in a bulk material the watery-component partial density determined in $\mathrm{g} / \mathrm{cm}^{3}$ is numerically equal to the dimensionless volumetric watery content.

By using the substitutions $\beta_{\mathrm{w}}(z)=b_{\mathrm{w}} \alpha_{\mathrm{w}}(z), F(z)=$ $\exp \left(-\int_{z_{0}}^{z} \alpha_{\mathrm{w}}\left(z^{\prime}\right) \mathrm{d} z^{\prime}\right)$ and $G(z)=\exp \left(-\int_{z_{0}}^{z} \alpha_{\mathrm{ds}}\left(z^{\prime}\right) \mathrm{d} z^{\prime}\right)$, Eq. (2) is reducible to the following first-order linear differential equation:

$$
\frac{\mathrm{d}}{\mathrm{d} z} F(z)-\left[\beta_{\mathrm{ds}}(z) / b_{\mathrm{w}}\right] F(z)+S(z) /\left[b_{\mathrm{w}} G(z)\right]=0 .
$$

The solution of Eq. (3) with respect to $F(z)$ is routinely obtainable under the natural boundary condition $F\left(z=z_{0}\right)=1$ and is valid for $z \in[0, \infty)$. Another expression of $F(z)$, for $z \in\left[0, z_{\mathrm{m}}\right]$, may also be obtained under the boundary condition $F\left(z=z_{\mathrm{m}}\right)=F_{\mathrm{m}}$. Then, in both the cases, the linear attenuation profile $\alpha_{\mathrm{w}}(z)$ is obviously expressible as

$$
\alpha_{\mathrm{w}}(z)=-\frac{\mathrm{d}}{\mathrm{d} z} \ln F(z) .
$$

The corresponding expressions obtained for $\alpha_{\mathrm{w}}(z)$ are:

$$
\begin{aligned}
& \alpha_{\mathrm{w}}(z)=-\beta_{\mathrm{ds}}(z) / b_{\mathrm{w}} \\
& +\frac{S(z) \exp \left(\int_{z_{0}}^{z}\left[\alpha_{\mathrm{ds}}\left(z^{\prime}\right)-\beta_{\mathrm{ds}}\left(z^{\prime}\right) / b_{\mathrm{w}}\right] \mathrm{d} z^{\prime}\right)}{b_{\mathrm{w}}-J_{1}(z)},
\end{aligned}
$$

and

$$
\begin{aligned}
& \alpha_{\mathrm{w}}(z)=-\beta_{\mathrm{ds}}(z) / b_{\mathrm{w}} \\
& +\frac{S(z) H\left(z_{0}, z_{\mathrm{m}}\right) \exp \left(-\int_{z}^{z_{\mathrm{m}}}\left[\alpha_{\mathrm{ds}}\left(z^{\prime}\right)-\beta_{\mathrm{ds}}\left(z^{\prime}\right) / b_{\mathrm{w}}\right] \mathrm{d} z^{\prime}\right)}{F_{\mathrm{m}} b_{\mathrm{w}}+H\left(z_{0}, z_{\mathrm{m}}\right) J_{2}(z)},
\end{aligned}
$$


where

$$
\begin{aligned}
& J_{1}(z)=\int_{z_{0}}^{z} \mathrm{~d} z^{\prime} S\left(z^{\prime}\right) \\
& \times \exp \left(\int_{z_{0}}^{z^{\prime}}\left[\alpha_{\mathrm{ds}}\left(z^{\prime \prime}\right)-\beta_{\mathrm{ds}}\left(z^{\prime \prime}\right) / b_{\mathrm{w}}\right] \mathrm{d} z^{\prime \prime}\right), \\
& J_{2}(z)=\int_{z}^{z_{\mathrm{m}}} \mathrm{d} z^{\prime} S\left(z^{\prime}\right) \\
& \quad \times \exp \left(-\int_{z^{\prime}}^{z_{\mathrm{m}}}\left[\alpha_{\mathrm{ds}}\left(z^{\prime \prime}\right)-\beta_{\mathrm{ds}}\left(z^{\prime \prime}\right) / b_{\mathrm{w}}\right] \mathrm{d} z^{\prime \prime}\right),
\end{aligned}
$$

and

$$
H\left(z_{0}, z_{\mathrm{m}}\right)=\exp \left(\int_{z_{0}}^{z_{\mathrm{m}}} \alpha_{\mathrm{ds}}\left(z^{\prime}\right) \mathrm{d} z^{\prime}\right) .
$$

Equations (5) and (6) represent in fact the backscattering coefficient of the absorbed substance, $\beta_{\mathrm{w}}(z)=$ $b_{\mathrm{w}} \alpha_{\mathrm{w}}(z)$, as the difference between the overall two-component backscattering coefficient and that of the absorbing medium, $\beta_{\mathrm{ds}}(z)$. When the absorbing dry medium consists of only one material, the value of $b_{\mathrm{ds}}(z) \equiv b_{\mathrm{ds}}=$ const so that $\beta_{\mathrm{ds}}(z)=b_{\mathrm{ds}} \alpha_{\mathrm{ds}}(z)$. Also, when the penetrating substance and absorbing medium consist of relatively light materials, such as, e.g., water, soil, aluminium, etc., $b_{\mathrm{w}}$ and $b_{\mathrm{ds}}$ will have practically the same value of $0.02[13]$. Then $\beta_{\mathrm{ds}}(z) / b_{\mathrm{w}}=\alpha_{\mathrm{ds}}(z)$ and the exponential factors in Eqs. (5), (6), (7a) and (7b) will be equal to unity.

The expressions of $\alpha_{\mathrm{w}}(z)$ given by Eqs. (5) and (6) are valid in general for both continuous and discontinuous dense media. The latter case concerns, e.g., the presence of air cavities or other inclusions in the way of the sensing photon beam. In this case, one should only exclude from consideration the discontinuity points of $\beta_{\mathrm{ds}}(z)\left(\alpha_{\mathrm{ds}}(z)\right)$ that are recognizable in the experimental profiles of $S(z)$ $\left(N_{T}\left(E_{\mathrm{bc}}, z, \Delta z\right)\right.$, see in [14-16]).

The relative rms error $\delta_{\mathrm{r} \alpha}=\left\langle\left[\widehat{\alpha}_{\mathrm{w}}(z)-\right.\right.$ $\left.\left.\alpha_{\mathrm{w}}(z)\right]^{2}\right\rangle^{1 / 2} / \alpha_{\mathrm{w}}(z)=\left\langle\left[\widehat{\rho}_{\mathrm{W}}(z)-\rho_{\mathrm{w}}(z)\right]^{2}\right\rangle / \rho_{\mathrm{w}}(z)$ and the relative bias error $B_{\mathrm{r} \alpha}(z)=\left[\left\langle\widehat{\alpha}_{\mathrm{w}}(z)\right\rangle-\alpha_{\mathrm{w}}(z)\right] / \alpha_{\mathrm{w}}(z)=$ $\left[\left\langle\widehat{\rho}_{\mathrm{w}}(z)\right\rangle-\rho_{\mathrm{w}}(z)\right] / \rho_{\mathrm{w}}(z)$ in the determination of $\alpha_{\mathrm{w}}(z)$ are estimated (see Appendix) by linear error propagation. The symbols ${ }^{\wedge}$ and $\langle$.$\rangle denote statistical estimate and$ ensemble average, respectively; $\widehat{\rho}_{\mathrm{w}}(z)=\widehat{\alpha}_{\mathrm{w}}(z) / \mu_{\mathrm{w}}$. The results obtained are

$$
\begin{gathered}
\delta_{\mathrm{r} \alpha 1}(z) \geq\left[1+\beta_{\mathrm{ds}}(z) / \beta_{\mathrm{w}}\right]\left\{N_{T}^{-1}\left(E_{\mathrm{bc}}, z, \Delta z\right)+N_{T J}^{-1}(z)\right. \\
\left.\times\left[\exp \left(\int_{z_{0}}^{z} \mathrm{~d} z^{\prime}\left[\alpha_{\mathrm{w}}\left(z^{\prime}\right)+\beta_{\mathrm{ds}}\left(z^{\prime}\right) / b_{\mathrm{w}}\right]\right)-1\right]^{2}\right\}^{1 / 2}
\end{gathered}
$$

and

$$
\begin{aligned}
& B_{\mathrm{r} \alpha 1}(z) \geq\left[1+\beta_{\mathrm{ds}}(z) / \beta_{\mathrm{w}}\right] N_{T J}^{-1 / 2}(z) \\
& \quad \times\left[\exp \left(\int_{z_{0}}^{z} \mathrm{~d} z^{\prime}\left[\alpha_{\mathrm{w}}\left(z^{\prime}\right)+\beta_{\mathrm{ds}}\left(z^{\prime}\right) / b_{\mathrm{w}}\right]\right)-1\right],
\end{aligned}
$$

for the expression of $\alpha_{\mathrm{w}}(z)$ given by Eq. (5), and

$$
\begin{aligned}
& \delta_{\mathrm{r} \alpha 2}(z) \geq\left[1+\beta_{\mathrm{ds}}(z) / \beta_{\mathrm{w}}\right]\left\{N_{T}^{-1}\left(E_{\mathrm{bc}}, z, \Delta z\right)\right. \\
& +N_{T J}^{-1}\left(z, z_{\mathrm{m}}\right) \\
& \quad \times\left[1-\exp \left(-\int_{z}^{z_{\mathrm{m}}}\left[\alpha_{\mathrm{w}}\left(z^{\prime}\right)\right.\right.\right. \\
& \left.\left.\left.\left.+\beta_{\mathrm{ds}}\left(z^{\prime}\right) / b_{\mathrm{w}}\right] \mathrm{d} z^{\prime}\right)\right]^{2}\right\}^{1 / 2},
\end{aligned}
$$

and

$$
\begin{aligned}
& B_{\mathrm{r} \alpha 2}(z) \geq\left[1+\beta_{\mathrm{ds}}(z) / \beta_{\mathrm{w}}\right] N_{T J}^{-1 / 2}\left(z, z_{\mathrm{m}}\right) \\
& \quad \times\left[1-\exp \left(-\int_{z}^{z_{\mathrm{m}}} \mathrm{d} z^{\prime}\left[\alpha_{\mathrm{w}}\left(z^{\prime}\right)+\beta_{\mathrm{ds}}\left(z^{\prime}\right) / b_{\mathrm{w}}\right]\right)\right],
\end{aligned}
$$

for the expression of $\alpha_{\mathrm{w}}(z)$ given by Eq. (6); $N_{T J}(z)=\sum_{i=0}^{\left(z-z_{0}\right) / \Delta z} N_{T}\left(E_{\mathrm{bc}}, z_{\mathrm{i}}, \Delta z\right), \quad$ and $N_{T J}\left(z, z_{\mathrm{m}}\right)=\sum_{i=\left(z-z_{0}\right) / \Delta z}^{\left(z_{\mathrm{m}}-z_{0}\right) / \Delta z} N_{T}\left(E_{\mathrm{bc}}, z_{\mathrm{i}}, \Delta z\right)$.

The analysis of Eqs. (8) and (9) shows that the relative rms error $\delta_{\mathrm{r} \alpha 1}(z)$ in the determination of $\alpha_{\mathrm{w}}$, involving the statistical and systematic errors, increases exponentially with $z$ and may have unacceptable value, even at large values of $N_{T}\left(E_{\mathrm{bc}}, z, \Delta z\right)$ and $N_{T J}(z)$.

At the same time, according to Eqs. (10) and (11), the relative rms error $\delta_{\mathrm{r} \alpha 2}(z)$ is mainly due to the statistical Poisson fluctuations. In this case, the statistical bias (Eq. (11)) is negligibly small at small values of $z$ where $N_{T J} \gg 1$. The increase of the bias with $z$ is relatively slow because in Eq. (11) the corresponding decrease of $N_{T J}\left(z, z_{\mathrm{m}}\right)$ is compensated for by the decrease of the numerator $1-\exp \left(-\int_{z}^{z_{\mathrm{m}}} \mathrm{d} z^{\prime}\left[\alpha_{\mathrm{w}}\left(z^{\prime}\right)+\beta_{\mathrm{ds}}\left(z^{\prime}\right) / b_{\mathrm{w}}\right]\right)$. Equations (8)-(11) show as well that in both the cases the error in the determination of the partial density profile of the absorbed substance is proportional to the penetrated amount of it and to the bulk density of the absorbing medium (through $\alpha_{\mathrm{w}}(z)$, and $\beta_{\mathrm{ds}}(z)$ and $\alpha_{\mathrm{ds}}(z)$, respectively); also, the error is inversely proportional to the sensing photon flux and the data acquisition time through $N_{T}$ and $N_{T J}$.

One more source of error in the determination of $\alpha_{\mathrm{w}}$ on the basis of Eq. (6) is the uncertainty $\Delta F_{\mathrm{m}}$ in the determination of the boundary value $F_{\mathrm{m}}$. According to the expression of Eq. (6), the influence of $\Delta F_{\mathrm{m}}$ on the measurement accuracy is more essential at large values of $z$ (near $z_{\mathrm{m}}$ ) and decreases with the decrease of $z$. Approximate values of $F_{\mathrm{m}}$ are obtainable by reducing Eq. (3), taking into account the expression of $F(z)$ and the fact that $\beta_{\mathrm{ds}}(z)=b_{\mathrm{ds}} \alpha_{\mathrm{ds}}(z)$ and $b_{\mathrm{ds}} \approx b_{\mathrm{w}}$, to the relation

$$
F(z) \approx S(z)\left[b_{\mathrm{w}} G(z)\right]^{-1}\left[\alpha_{\mathrm{w}}(z)+\alpha_{\mathrm{ds}}(z)\right]^{-1} .
$$

When $\alpha_{\mathrm{w}}(z) \ll \alpha_{\mathrm{ds}}(z)$, e.g., at relatively low moisture in soil, the relation (12) leads to the following approximate boundary condition:

$$
F_{\mathrm{m}}=F\left(z=z_{\mathrm{m}}\right) \approx S\left(z_{\mathrm{m}}\right)\left[b_{\mathrm{w}} \alpha_{\mathrm{ds}}\left(z_{\mathrm{m}}\right) G\left(z_{\mathrm{m}}\right)\right]^{-1} .
$$

In the opposite case, when $\alpha_{\mathrm{w}}(z) \gg \alpha_{\mathrm{ds}}(z)$ (high- 
-porosity soil with high water content), the neglect of the term $\left[\beta_{\mathrm{ds}}(z) / b_{\mathrm{w}}\right] F(z)$ in Eq. (3) leads to another approximate boundary condition

$$
F_{\mathrm{m}} \approx 1-\int_{z_{0}}^{z_{\mathrm{m}}} \mathrm{d} z^{\prime} S\left(z^{\prime}\right)\left[b_{\mathrm{w}} G\left(z^{\prime}\right)\right]^{-1} .
$$

At last, in the "moderate case", when $\alpha_{\mathrm{w}}(z) \sim \alpha_{\mathrm{ds}}(z)$, from (12) it approximately follows that:

$$
F_{\mathrm{m}} \approx S\left(z_{\mathrm{m}}\right)\left[2 b_{\mathrm{w}} \alpha_{\mathrm{ds}}\left(z_{\mathrm{m}}\right) G\left(z_{\mathrm{m}}\right)\right]^{-1} .
$$

The applicability of the approximate conditions (13)-(15) is numerically estimated and confirmed in the following Sect. 4 .

\section{Simulations}

The photon count fluctuations are simulated under the assumption that they have Poisson statistics. Then the only parameter of importance is given by the graydar profile $N_{T}\left(E_{\mathrm{bc}}, z, \Delta z\right)$. At given models of the LOS profiles $\alpha(z)$ and $\beta(z)$ of the extinction and backscattering coefficients, the graydar profile is calculated according to Eq. (1). Then its realizations are obtained by using a Poisson random-number generator.

Four types of soil, say $A, B, C$, and $D$, are considered as probed media (see Table). Their elemental compositions are described in Refs. [24-27], respectively. By using data from [22, 23], the corresponding values of $\langle Z / A\rangle$ and the two-way mass attenuation coefficients $\mu=\mu_{\mathrm{f}}+\mu_{\mathrm{b}}$ are calculated, where $\mu_{\mathrm{f}}$ and $\mu_{\mathrm{b}}$ are the forward and backward components for photon energies $E_{\mathrm{f}}=511 \mathrm{keV}$ and $E_{\mathrm{bc}}=170.33 \mathrm{keV}$. Expectedly, the values obtained of $\mu$ and $\langle Z \mid A\rangle$ are practically coincident (Table). So, for estimations concerning all types of soil, independently of their elemental composition, the average values of $\mu=0.224 \mathrm{~cm}^{2} / \mathrm{g}$ and $\langle Z / A\rangle=0.508$ can be used. The graydar ratios are also coincident: $\beta_{\mathrm{ds}}=\beta_{\mathrm{w}}=0.02$ for all the soils.

The simulated experimental parameters are chosen to be: $z_{0}=10 \mathrm{~cm}, \Delta z=1 \mathrm{~cm}, r_{\mathrm{d}}=2.5 \mathrm{~cm}, r_{\mathrm{c}}=0.1 \mathrm{~cm}$, $E_{\mathrm{f}}=511 \mathrm{keV}, E_{\mathrm{bc}}=170.33 \mathrm{keV}$, and $\eta(z) \equiv 1 ; q_{0}$ and $T$ may be varied. It is also assumed that the angular divergence of the incident photon beam is $\alpha_{\mathrm{d}}=$ $r_{\mathrm{c}} / h=0.01745 \mathrm{rad}$, i.e. $1^{\circ} ;-h=-5.73 \mathrm{~cm}$ is the LOS position of the source of gamma photons. Then, if the positron-emission activity of the radionuclide employed is $a=300 \mathrm{mCi}$, the mean sensing photon flux will be $q_{0}=3.7 \times 10^{7} a \alpha_{\mathrm{d}}^{2} / 2 \approx 1.68 \times 10^{6} \mathrm{~s}^{-1}$. The corresponding transversal resolution radius $r_{\mathrm{t}} \sim \alpha_{\mathrm{d}}(z+h)[\mathrm{cm}]$ will vary from $r_{\mathrm{t}} \approx 0.27 \mathrm{~cm}$, at $z=z_{0}$, to $r_{\mathrm{t}} \approx 1 \mathrm{~cm}$, at $z=50 \mathrm{~cm}$. The angle of acceptance $\alpha_{\mathrm{a}} \sim r_{\mathrm{d}} / z$ comprised by the graydar receiving aperture will vary from $\alpha_{\mathrm{a}} \approx 0.25 \mathrm{rad}$, at $z=z_{0}$, to $\alpha_{\mathrm{a}} \approx 0.042 \mathrm{rad}$, at $z=50 \mathrm{~cm}$. At these values of $\alpha_{\mathrm{a}}$ the energy of the detected once-only-backscattered (signal) photons, within the solid angle $\Omega_{\mathrm{S}}=\pi \alpha_{\mathrm{a}}^{2}$, will vary with the angle of incidence with maximum about $1 \%$. Also, the largest difference $=z\left(\cos ^{-1} \alpha_{\mathrm{a}}-1\right)$ between the return path of a registered signal photon and the outbound path of the corresponding sensing photon will have a maximum value of $\approx 3 \mathrm{~mm}$ at $z=z_{0}$. This would lead to an error in the
TABLE

Elemental compositions of the four considered types of soil [24-27] and corresponding calculated values of the two-way mass attenuation coefficient $\mu=\mu_{\mathrm{f}}+\mu_{\mathrm{b}}$

\begin{tabular}{|c|c|c|c|c|}
\hline & Soil $A$ & Soil $B$ & Soil $C$ & Soil $D$ \\
\hline Element & \multicolumn{4}{|c|}{ Fraction by weight [\%] } \\
\hline $\mathrm{H}$ & 2.8 & 2.2 & 2.1 & 0.2100 \\
\hline $\mathrm{Li}$ & & & & 0.0013 \\
\hline $\mathrm{B}$ & & & & 0.0013 \\
\hline $\mathrm{C}$ & 14.4 & & 1.6 & 0.1950 \\
\hline $\mathrm{N}$ & & & & 0.0032 \\
\hline $\mathrm{O}$ & 49.7 & 57.5 & 57.7 & 53.0200 \\
\hline $\mathrm{F}$ & & & & 0.0046 \\
\hline $\mathrm{Na}$ & 0.8 & & & 0.2450 \\
\hline $\mathrm{Mg}$ & & & & 0.0881 \\
\hline $\mathrm{Al}$ & 8.9 & 8.5 & 5 & 1.0960 \\
\hline $\mathrm{Si}$ & 21.3 & 26.2 & 27.1 & 43.7600 \\
\hline $\mathrm{P}$ & & & & 0.0128 \\
\hline $\mathrm{Cl}$ & & & & 0.0108 \\
\hline $\mathrm{K}$ & 0.6 & & 1.3 & 0.2250 \\
\hline $\mathrm{Ca}$ & 0.5 & & 4.1 & 0.1180 \\
\hline $\mathrm{Sc}$ & & & & 0.0005 \\
\hline $\mathrm{Ti}$ & & & & 0.1228 \\
\hline $\mathrm{V}$ & & & & 0.0035 \\
\hline $\mathrm{Cr}$ & & & & 0.0029 \\
\hline Mn & & & & 0.0151 \\
\hline $\mathrm{Fe}$ & 1 & 5.6 & 1.1 & 0.7240 \\
\hline Co & & & & 0.0007 \\
\hline $\mathrm{Ni}$ & & & & 0.0009 \\
\hline $\mathrm{Cu}$ & & & & 0.0012 \\
\hline $\mathrm{Rb}$ & & & & 0.0058 \\
\hline $\mathrm{Y}$ & & & & 0.0035 \\
\hline $\mathrm{Zr}$ & & & & 0.0545 \\
\hline $\mathrm{Ba}$ & & & & 0.0540 \\
\hline $\mathrm{Nd}$ & & & & 0.0063 \\
\hline $\mathrm{Sm}$ & & & & 0.0012 \\
\hline Hf & & & & 0.0029 \\
\hline $\mathrm{Pb}$ & & & & 0.0050 \\
\hline $\mathrm{Bi}$ & & & & 0.0051 \\
\hline $\mathrm{Th}$ & & & & 0.0037 \\
\hline$\mu=\mu_{\mathrm{f}}+\mu_{\mathrm{b}},\left[\mathrm{cm}^{2} / \mathrm{g}\right]$ & 0.2246 & 0.225 & 0.2242 & 0.2207 \\
\hline$\langle Z / A\rangle$ & 0.511 & 0.507 & 0.5084 & 0.5036 \\
\hline
\end{tabular}
and the mean ratio $\langle Z / A\rangle$.

positioning of the acts of backscattering along the LOS that does not exceed $1.5 \mathrm{~mm}$ at $z=z_{0}$ and decreases with $z$.

The relative Doppler broadening $\Delta E_{\mathrm{Dr}}=\Delta E_{\mathrm{D}} / E_{\mathrm{bc}}$ should be about $5 \%$. It is determined in fact by the strongly prevailing elements in soil such as ${ }_{8} \mathrm{O},{ }_{14} \mathrm{Si}$, 
${ }_{13} \mathrm{Al}$, and ${ }_{26} \mathrm{Fe}$ whose fractions by weight are, respectively, about $50 \%-60 \%, 20 \%-40 \%, 5 \%-9 \%$, and $1 \%-6 \%$ (see Table). On the basis of some available experimental data [19] one may deduce for instance that at $E_{\mathrm{f}}=511 \mathrm{keV}$ and $E_{\mathrm{bc}}=170.33 \mathrm{keV}, \Delta E_{\mathrm{Dr}} \approx 5 \%$ for aluminium. For oxygen the Doppler broadening effect should be still weaker. An upper estimate of $\Delta E_{\mathrm{Dr}}$ of the same order $(\approx 3.2 \%-6.4 \%)$ is also obtainable by calculating in momentum approximation the contribution to the Doppler broadening of $K$-shell electrons in free ${ }_{13} \mathrm{Al},{ }_{14} \mathrm{Si}$, and ${ }_{26} \mathrm{Fe}$ atoms (e.g., $\left.[28,29]\right)$. Thus, to avoid the loss of signal photons one should use gamma ray detectors with $\pm 5 \%( \pm 8.5 \mathrm{keV})$ wide energy-discrimination window around the central energy of $170 \mathrm{keV}$. Such an energy resolution of detecting $170 \mathrm{keV}$ gamma photons is achievable, e.g., by lanthanium-based scintillation detectors [30], but at the expense of lower temporal resolution and timing accuracy of the photon count pulses. The contemporary scientifical efforts in this field are directed just to the development of very fast, high energy resolution gamma-photon detectors [31-33].

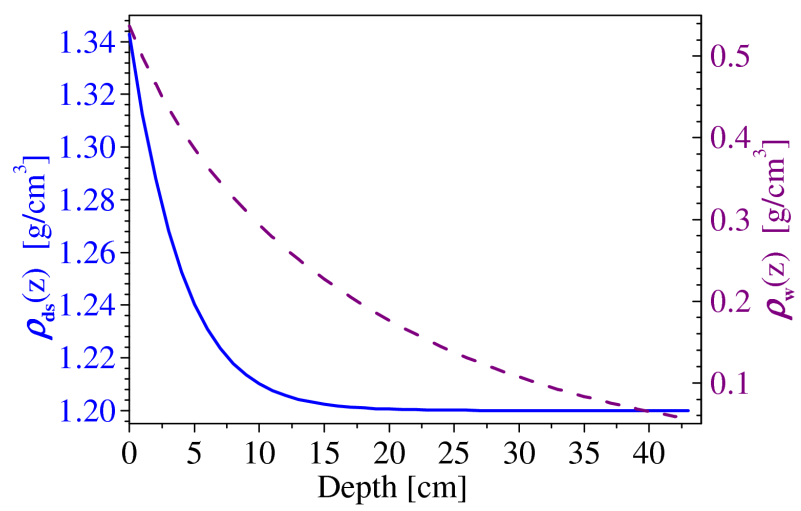

Fig. 2. Models of in-depth profiles of the bulk density of soil $\rho_{\mathrm{ds}}(z)$ (solid curve, left ordinate) and the partial density of water in soil $\rho_{\mathrm{w}}(z)$ (dashed curve, right ordinate).

The bulk density of soil and the partial density of water in soil are modeled, respectively, as varying along the LOS according to the laws

$$
\begin{aligned}
& \rho_{\mathrm{ds}}(z)=\rho_{\mathrm{n}}\left[1+\left(z / z_{\mathrm{p}}\right) \exp \left(-z / z_{\mathrm{p}}\right)\right], \\
& \text { and } \\
& \rho_{\mathrm{w}}(z)=A \rho_{\mathrm{ds}}(z) \exp \left(-\left(z-z_{0}\right) / z_{\mathrm{w}}\right),
\end{aligned}
$$

where $\rho_{\mathrm{n}}, A, z_{\mathrm{p}}$, and $z_{\mathrm{w}}$ are characteristic parameters that may take different values. In the simulations performed it is assumed that $z_{\mathrm{p}}=3 \mathrm{~cm}, A=0.4, z_{0}=10 \mathrm{~cm}$ and $z_{\mathrm{w}}=20 \mathrm{~cm} ; \rho_{\mathrm{n}}$ was varied between $1 \mathrm{~g} / \mathrm{cm}^{3}$ and $1.3 \mathrm{~g} / \mathrm{cm}^{3}$. At this choice of the parameters, the ratio $C(z)=\rho_{\mathrm{w}}(z) / \rho_{\mathrm{ds}}(z)=A \exp \left(-\left(z-z_{0}\right) / z_{\mathrm{w}}\right)$ characterizing the contrast between the water content and the dry-soil content varies from $C=A=0.4$ at $z-z_{0}=0$, through $C=0.15$ at $z-z_{0}=20 \mathrm{~cm}$, to $C=0.05$ at $z-z_{0}=40 \mathrm{~cm}$. The models of $\rho_{\mathrm{ds}}(z)$ and $\rho_{\mathrm{w}}(z)$ used in the simulations for $\rho_{\mathrm{n}}=1.2 \mathrm{~g} / \mathrm{cm}^{3}$ are represented in

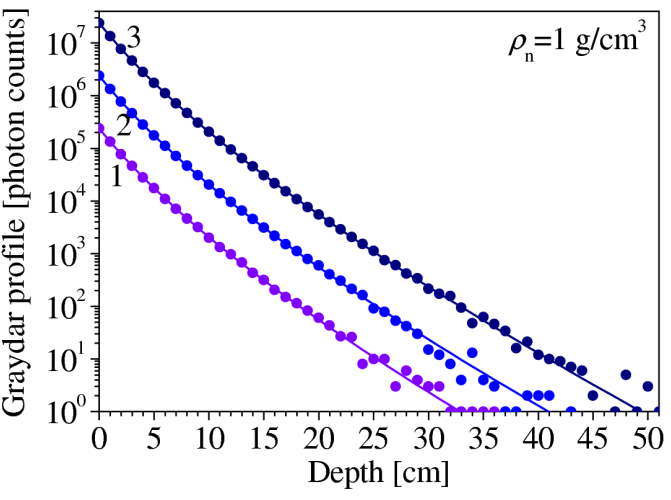

Fig. 3. Realizations of the graydar profiles (circles) compared with the expected ones given by solid curves for values of $q_{0} T$ equal to $1.68 \times 10^{8}(1), 1.68 \times 10^{9}(2)$, and $1.68 \times 10^{10}(3)$.

Fig. 2.

Simulating the procedure of recovering the profile $\rho_{\mathrm{w}}(z)$ under the Poisson noise conditions shows that as expected, due to stronger accumulated signal and thus to higher measurement accuracy, the depth of accurate sensing increases with decreasing the soil density $\left(\rho_{\mathrm{n}}\right)$ and increasing the sensing photon flux and the measurement time. This is well seen in Fig. 3, and especially in Fig. 4 where some recovered by algorithms (5) and (6) in-depth distributions $\rho_{\mathrm{w}}(z)$ are compared with the premised model given by solid curve. The dashed curves are obtained by fitting the data obtained by algorithm (6). The exact boundary value is used of $F_{\mathrm{m}}=F\left(z=z_{\mathrm{m}}\right)$ at $z_{\mathrm{m}}=49 \mathrm{~cm}$ (Fig. 4a), $42 \mathrm{~cm}$ (Fig. 4b), and $58 \mathrm{~cm}$ (Fig. 4c). The negative sign of some of the recovered values of $\rho_{\mathrm{wr}}(z)$ given in Figs. $4 \mathrm{a}-\mathrm{c}$ has no realistic sense at all, but in fact it is realistically interpretable as due to underestimating the two-component, moist-soil backscattering coefficient $\beta_{\mathrm{ds}}(z)+\beta_{\mathrm{w}}(z)$ (see Eqs. (5) and (6)). Therefore the fitting procedures performed are quite reasonable. The behaviour of the relative rms error in the determination of $\rho_{\mathrm{w}}(z)$ by algorithms (5) and (6) is shown in Fig. 5 . It confirms the theoretical expectation (see the discussion below Eq. (11)) that algorithm (6) should be more accurate than algorithm (5). So, it is seen that algorithm (5) ensures an accurate retrieval to depths of about $17 \mathrm{~cm}$, at $\rho_{\mathrm{n}}=1 \mathrm{~g} / \mathrm{cm}^{3}$ and $q_{0} T=1.68 \times 10^{9}$ (Fig. $\left.4 \mathrm{a}\right), 14 \mathrm{~cm}$, at $\rho_{\mathrm{n}}=1.3 \mathrm{~g} / \mathrm{cm}^{3}$ and $q_{0} T=1.68 \times 10^{9}$ (Fig. $\left.4 \mathrm{~b}\right)$, and $25 \mathrm{~cm}$, at $\rho_{\mathrm{n}}=1 \mathrm{~g} / \mathrm{cm}^{3}$ and $q_{0} T=1.68 \times 10^{10}$ (Fig. 4c). With increasing the depth above the indicated values an unacceptable bias takes place. At the same time, the partial density profile $\rho_{\mathrm{wr}}(z)$ recovered by using algorithm (6) is unbiased and closely coincident with the true one to depths of $26 \mathrm{~cm}, 23 \mathrm{~cm}$, and $32 \mathrm{~cm}$, respectively. Moreover, a fit to the data obtained for $\rho_{\mathrm{wr}}(z)$ practically coincides with the true profile to depths of about $40 \mathrm{~cm}$ in Fig. $4 \mathrm{a}, 33 \mathrm{~cm}$ in Fig. $4 \mathrm{~b}$, and $50 \mathrm{~cm}$ in Fig. 4c. The corresponding mean relative deviation 

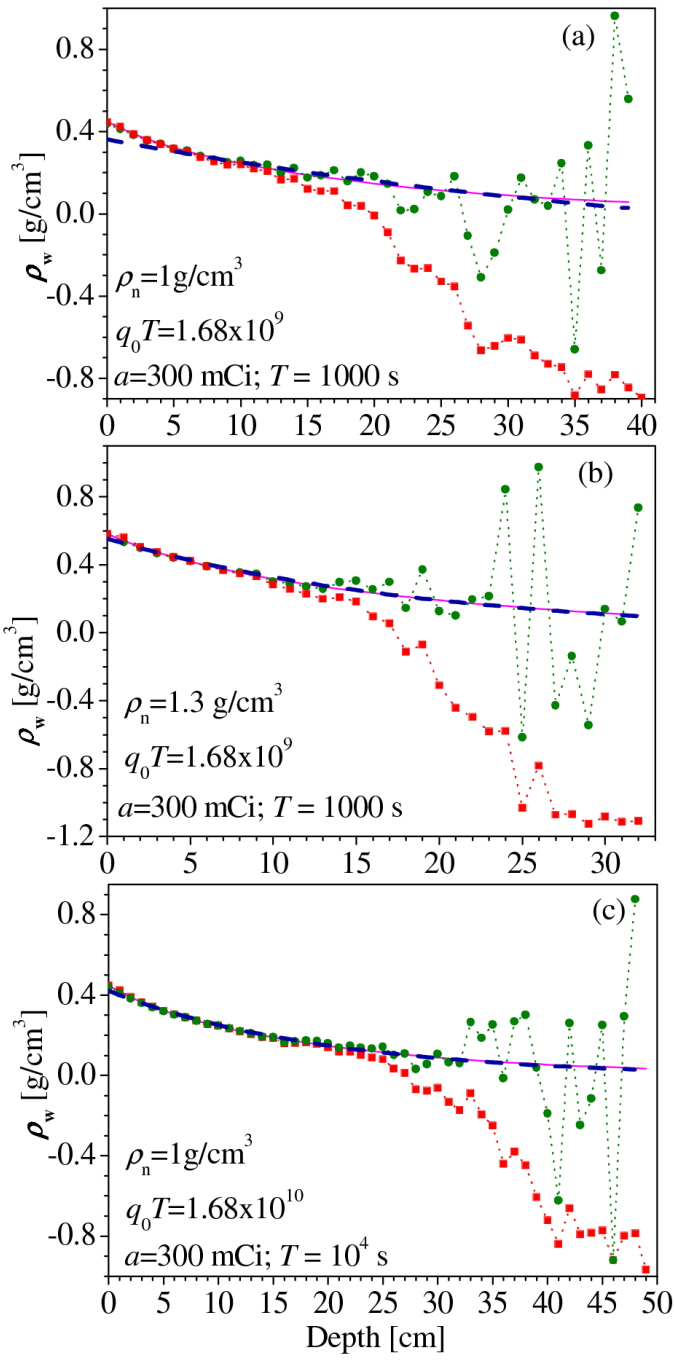

Fig. 4. In-depth distributions $\rho_{\mathrm{wr}}(z)$ of the partial density of the absorbed water (compared with the true one given by solid curve) recovered using algorithms (5) (squares) and (6) (circles) at different values of $\rho_{\mathrm{n}}$ and $q_{0} T$. The dashed curves are obtained by fitting the data recovered by algorithm (6).

$d_{\mathrm{r} \rho}(0, z)=\left[\int_{z_{0}}^{z}\left|\rho_{\mathrm{wf}}(z)-\rho_{\mathrm{w}}(z)\right| \mathrm{d} z\right] /\left[\int_{z_{0}}^{z} \rho_{\mathrm{w}}(z) \mathrm{d} z\right]$ of the fitted profiles $\rho_{\mathrm{wf}}(z)$ in Figs. 4a,b and c are estimated to be equal to $0.085\left(8.5 \%, z-z_{0}=39 \mathrm{~cm}\right), 0.023(2.3 \%$, $\left.z-z_{0}=32 \mathrm{~cm}\right)$, and $0.03\left(3 \%, z-z_{0}=48 \mathrm{~cm}\right)$.

Figure 6 illustrates the performance of algorithm (6) in the case of using the approximate boundary condition (13) for $F_{\mathrm{m}}$. The value of $z_{\mathrm{m}}$ is chosen to be $49 \mathrm{~cm}$, that is, the depth $z_{\mathrm{m}}-z_{0}=39 \mathrm{~cm}$. As it is seen, the result from the retrieval is near that obtained for the true value of $F_{\mathrm{m}}$ (Fig. 4a). Thus, the use of the approximate boundary condition (13) turns out to be acceptable and expedient. The use in the simulations of the other approximate boundary conditions, (14) and (15), also ensures acceptable accuracy of recovering the moisture profile $\rho_{\mathrm{w}}(z)$. This is seemingly due, according to

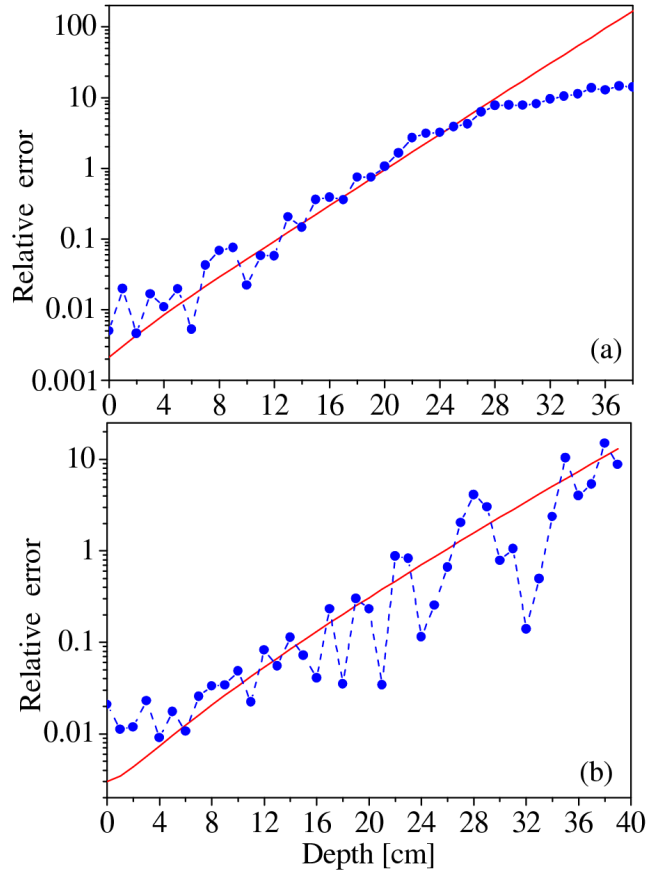

Fig. 5. Theoretically estimated rms relative error (solid curves) in the determination of the partial density $\rho_{\mathrm{w}}(z)$ of the absorbed water, using algorithms (5) (a) and (6) (b), compared with the absolute values $d_{\mathrm{r} \rho}(z)$ (circles) of the relative deviations of the recovered profile $\rho_{\mathrm{wr}}(z)$ for $\rho_{\mathrm{n}}=1 \mathrm{~g} / \mathrm{cm}^{3} ; d_{\mathrm{r} \rho}(z)=\mid \rho_{\mathrm{wr}}(z)-$ $\rho_{\mathrm{w}}(z) \mid / \rho_{\mathrm{w}}(z)$. The experimental conditions supposed and the results illustrated here correspond to those concerning Fig. 4a.

the character of Eq. (6), to the low sensitivity of this algorithm to uncertainties in the determination of the boundary value $F_{\mathrm{m}}$.

As a whole, the results from the simulations conducted show that the in-depth soil moisture profile can be accurately measured using graydar to depths of 40-50 cm, depending on the bulk density of soil, the sensing photon flux, and the measurement (data acquisition) time. It turns out as well that the second of the retrieval algorithms, algorithm (6), would ensure a higher sensing accuracy compared to algorithm (5), and correspondingly a larger depth of accurate sensing. The approximate boundary conditions concerning algorithm (6) are shown to not lead to noticeable lowering of the retrieval accuracy. Mention at last that the simulations performed illustrate the possibility of measuring the soil moisture profile at a contrast, with respect to the dry-soil profile, of 0.05 at a depth of $40 \mathrm{~cm}$.

\section{Summary}

In the present work the possibility is investigated of determining using graydar the in-depth profile of the partial density of a substance absorbed by a medium with known bulk density distribution. On the basis of the graydar 


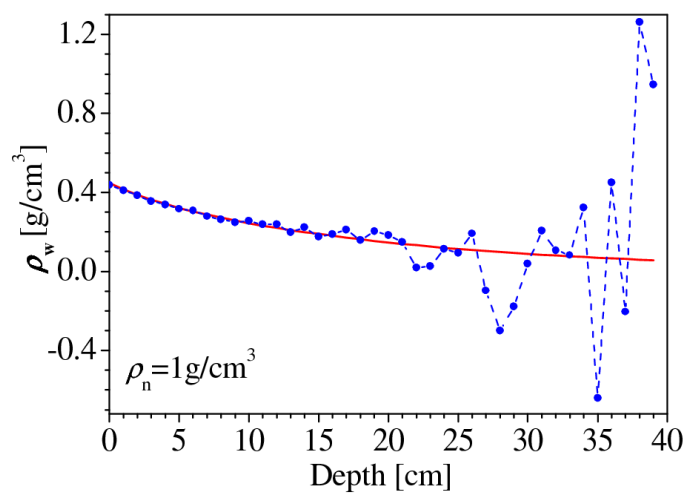

Fig. 6. In-depth distribution $\rho_{\mathrm{wr}}(z)$ of the partial density of absorbed water recovered by algorithm (6) in the case of using an approximate value of $F_{\mathrm{m}}$. The model supposed of $\rho_{\mathrm{w}}(z)$ is given by solid curve; $q_{0} T=$ $1.68 \times 10^{9}$.

equation two inverse algorithms have been derived for recovering the in-depth partial-density profile of the absorbed substance on the basis of the known bulk-density profile of the absorbing medium, the known parameters of the experimental setup, and the experimentally determinable graydar profile. Analytical lower estimates of the corresponding Poisson-noise-due retrieval errors and approximate "internal" boundary conditions underlying the performance of the "second" of the algorithms have also been obtained. To examine and illustrate the algorithm performance, the recovering procedures have been numerically simulated, taking into account the Poisson fluctuations of the graydar signal. The important for applications case has been considered when the absorbed substance is water, and the absorbing medium is soil. The results obtained are in agreement with the theoretical results and conclusions. So, they show that the error of sensing of the moisture profile is proportional to the bulk density of soil, and is inversely proportional to the sensing photon flux and the data-acquisition time. Besides, the "second" algorithm (6) turns out to be more accurate indeed than the "first" algorithm (5), thus allowing one to achieve a larger depth of accurate sensing. The simulations also show that the use of the approximate boundary conditions concerning algorithm (6) does not lead to noticeable lowering of the retrieval accuracy. The soil moisture profile could be accurately determined to depths of $40-50 \mathrm{~cm}$, at a resolution scale of $1 \mathrm{~cm}$ and measurement time of, e.g., $1000 \mathrm{~s}$ when $q_{0}=1.68 \times 10^{6} \mathrm{~s}^{-1}$. The detectable water-to-dry-soil density contrast could be as small as $5 \%$ at a depth of $40 \mathrm{~cm}$. The three-dimensional moisture distribution is obtainable by laterally scanning the graydar LOS or by using simultaneously a set of parallel sensing beams.

The realization of the discussed here graydar approach would require experimental equipment ensuring fine-enough discrimination of the instants of emission of the sensing photons and the arrival times and energy of the backscattered photons. Such an equipment may be available soon as a result of the contemporary progress in the development of fast high-energy-resolution detectors and analyzers of gamma photons [30-32].

\section{Acknowledgments}

This research was partially supported by the Bulgarian National Science Fund under project No. DO-02-107.

\section{Appendix}

At a given experimental realization $\widehat{N}_{T}\left(E_{\mathrm{bc}}, z, \Delta z\right)$ of the graydar profile $N_{T}\left(E_{\mathrm{bc}}, z, \Delta z\right)$, according to Eqs. (5) and $(6)$, the corresponding estimate $\widehat{\alpha_{\mathrm{w}}}(z)$ of the profile $\alpha_{\mathrm{w}}(z)$ is given by the expression

$$
\begin{aligned}
& \widehat{\alpha_{\mathrm{w}}}(z)=-\beta_{\mathrm{ds}}(z) / b_{\mathrm{w}} \\
& +\frac{\hat{S}(z) \exp \left(\int_{z_{0}}^{z}\left[\alpha_{\mathrm{ds}}\left(z^{\prime}\right)-\beta_{\mathrm{ds}}\left(z^{\prime}\right) / b_{\mathrm{w}}\right] \mathrm{d} z^{\prime}\right)}{b_{\mathrm{w}}-\widehat{J_{1}}(z)},
\end{aligned}
$$

following from Eq. (5), or the expression

$$
\begin{aligned}
& \widehat{\alpha_{\mathrm{w}}}(z)=-\beta_{\mathrm{ds}}(z) / b_{\mathrm{w}} \\
& +\frac{\hat{S}(z) H\left(z_{0}, z_{\mathrm{m}}\right) \exp \left(-\int_{z}^{z_{\mathrm{m}}}\left[\alpha_{\mathrm{ds}}\left(z^{\prime}\right)-\beta_{\mathrm{ds}}\left(z^{\prime}\right) / b_{\mathrm{w}}\right] \mathrm{d} z^{\prime}\right)}{F_{\mathrm{m}} b_{\mathrm{w}}+H\left(z_{0}, z_{\mathrm{m}}\right) \widehat{J_{2}}(z)},
\end{aligned}
$$

following from Eq. (6), where

$$
\begin{aligned}
& \hat{S}(z)=\widehat{N}_{T}\left(E_{\mathrm{bc}}, z, \Delta z\right) / W(z) \\
& W(z)=d q_{0} \Delta z T z^{-2} \eta(z) \\
& \widehat{J_{1}}(z)=\int_{z_{0}}^{z} \mathrm{~d} z^{\prime} \hat{S}\left(z^{\prime}\right) \\
& \quad \times \exp \left(\int_{z_{0}}^{z^{\prime}}\left[\alpha_{\mathrm{ds}}\left(z^{\prime \prime}\right)-\beta_{\mathrm{ds}}\left(z^{\prime \prime}\right) / b_{\mathrm{w}}\right] \mathrm{d} z^{\prime \prime}\right) \\
& \widehat{J_{2}}(z)=\int_{z}^{z_{\mathrm{m}}} \mathrm{d} z^{\prime} \hat{S}\left(z^{\prime}\right) \\
& \quad \times \exp \left(-\int_{z^{\prime}}^{z_{\mathrm{m}}}\left[\alpha_{\mathrm{ds}}\left(z^{\prime \prime}\right)-\beta_{\mathrm{ds}}\left(z^{\prime \prime}\right) / b_{\mathrm{w}}\right] \mathrm{d} z^{\prime \prime}\right) .
\end{aligned}
$$

For estimating the relative rms error $\delta_{\mathrm{r} \alpha}=\left\langle\left[\widehat{\alpha_{\mathrm{W}}}(z)-\right.\right.$ $\left.\left.\alpha_{\mathrm{w}}(z)\right]^{2}\right\rangle^{1 / 2} / \alpha_{\mathrm{w}}(z)$ in the determination of $\alpha_{\mathrm{w}}(z)$, the estimate $\widehat{\alpha_{\mathrm{w}}}$ may be considered as a function of two random variables: $\hat{S}(z)$ and $\widehat{J_{1}}(z)$ [or $\widehat{J_{2}}(z)$ ] when given by Eq. (18) [or by Eq. (19)]. The random variables obviously obey the relations $\langle\hat{S}(z)\rangle=S(z)$, and $\left\langle\widehat{J_{1,2}}(z)\right\rangle=J_{1,2}(z)$. By linear error transfer based on Eqs. (18) and (19) the following general expressions of $\delta_{\mathrm{r} \alpha}(z)$ are obtained, respectively:

$$
\begin{aligned}
& \delta_{\mathrm{r} \alpha 1}(z)=\left\{\left[\frac{\partial \ln \alpha_{\mathrm{w}}(z)}{\partial S(z)}\right]^{2} \operatorname{Var} \hat{S}(z)\right. \\
& \left.+\left[\frac{\partial \ln \alpha_{\mathrm{w}}(z)}{\partial J_{1}(z)}\right]^{2} \operatorname{Var} \widehat{J_{1}}(z)\right\}^{1 / 2}=\left(1+\beta_{\mathrm{ds}} / \beta_{\mathrm{w}}\right)
\end{aligned}
$$




$$
\times\left\{[S(z)]^{-2} \operatorname{Var} \hat{S}(z)+\left[b_{\mathrm{w}}-J_{1}(z)\right]^{-2} \operatorname{Var} \widehat{J}_{1}(z)\right\}^{1 / 2},
$$

and

$$
\begin{aligned}
& \delta_{\mathrm{r} \alpha 2}(z)=\left\{\left[\frac{\partial \ln \alpha_{\mathrm{w}}(z)}{\partial S(z)}\right]^{2} \operatorname{Var} \hat{S}(z)\right. \\
& \left.\quad+\left[\frac{\partial \ln \alpha_{\mathrm{w}}(z)}{\partial J_{2}(z)}\right]^{2} \operatorname{Var} \widehat{J_{2}}(z)\right\}^{1 / 2} \\
& \quad=\left\{\left(1+\beta_{\mathrm{ds}} / \beta_{\mathrm{w}}\right)[S(z)]^{-2} \operatorname{Var} \hat{S}(z)\right. \\
& \quad+\left[H\left(z_{0}, z_{\mathrm{m}}\right)\right]^{2}\left[F_{\mathrm{m}} b_{\mathrm{w}}+H\left(z_{0}, z_{\mathrm{m}}\right) J_{2}(z)\right]^{-2} \\
& \left.\quad \times \operatorname{Var} \widehat{J_{2}}(z)\right\}^{1 / 2},
\end{aligned}
$$

where $\operatorname{Var} Y(z)=\left\langle[Y(z)-\langle Y(z)\rangle]^{2}\right\rangle$.

Let us further concretely perform a more detailed estimation of $\delta_{\mathrm{r} \alpha 1}(z)$. So, on the basis of Eq. (1) it follows that:

$$
[S(z)]^{-2} \operatorname{Var} \hat{S}(z)=N_{T}^{-1}\left(E_{\mathrm{bc}}, z, \Delta z\right) .
$$

Also, by using Eqs. (2) and (7a), the relation

$$
b_{\mathrm{w}}-J_{1}(z)=b_{\mathrm{w}} \exp \left(-\int_{z_{0}}^{z} \mathrm{~d} z^{\prime}\left[\alpha_{\mathrm{w}}\left(z^{\prime}\right)+\beta_{\mathrm{ds}}\left(z^{\prime}\right) / b_{\mathrm{w}}\right]\right)
$$

is derived. On the basis of Eq. (7a), the following expression for $\operatorname{Var} \widehat{J_{1}}(z)$ can be written:

$$
\begin{aligned}
& \operatorname{Var} \widehat{J_{1}}(z) \\
& \quad=\iint_{z_{0}}^{z} \mathrm{~d} z^{\prime} \mathrm{d} z^{\prime \prime} \operatorname{Var}^{1 / 2} \hat{S}\left(z^{\prime}\right) \operatorname{Var}^{1 / 2} \hat{S}\left(z^{\prime \prime}\right) K\left(z^{\prime}, z^{\prime \prime}\right) \\
& \quad \times \exp \left(\int_{z_{0}}^{z^{\prime}} \mathrm{d} \xi\left[\alpha_{\mathrm{ds}}(\xi)-\beta_{\mathrm{ds}}(\xi) / b_{\mathrm{w}}\right]\right. \\
& \left.\quad+\int_{z_{0}}^{z^{\prime \prime}} \mathrm{d} \xi\left[\alpha_{\mathrm{ds}}(\xi)-\beta_{\mathrm{ds}}(\xi) / b_{\mathrm{w}}\right]\right)
\end{aligned}
$$

where $K\left(z^{\prime}, z^{\prime \prime}\right)=\operatorname{Cov}_{S}\left(z^{\prime}, z^{\prime \prime}\right) /\left[\operatorname{Var} \hat{S}\left(z^{\prime}\right) \operatorname{Var} \hat{S}\left(z^{\prime \prime}\right)\right]^{1 / 2}$ is the correlation coefficient of $\hat{S}(z)$, and $\operatorname{Cov}_{S}\left(z^{\prime}, z^{\prime \prime}\right)=$ $\left\langle\left[\hat{S}\left(z^{\prime}\right)-S\left(z^{\prime}\right)\right]\left[\hat{S}\left(z^{\prime \prime}\right)-S\left(z^{\prime \prime}\right)\right]\right\rangle$ is the covariance of $\hat{S}(z)$. After the change of variables $\zeta=\left(z^{\prime}+z^{\prime \prime}\right) / 2$ and $\eta=z^{\prime}-z^{\prime \prime}$, Eq. (25) acquires the form

$$
\begin{aligned}
& \operatorname{Var} \widehat{J}_{1}(z)=\int_{-\left(z-z_{0}\right)}^{z-z_{0}} \mathrm{~d} \eta \int_{z_{0}+|\eta / 2|}^{z-|\eta / 2|} \mathrm{d} \zeta \\
& \quad \times[\operatorname{Var} \hat{S}(\zeta+\eta / 2) \operatorname{Var} \hat{S}(\zeta-\eta / 2)]^{1 / 2} \\
& \quad \times K(\zeta+\eta / 2, \zeta-\eta / 2) \\
& \quad \times \exp \left(\int_{z_{0}}^{\zeta+\eta / 2}\left[\alpha_{\mathrm{ds}}(\xi)-\beta_{\mathrm{ds}}(\xi) / b_{\mathrm{w}}\right] \mathrm{d} \xi\right.
\end{aligned}
$$

$$
\left.+\int_{z_{0}}^{\zeta-\eta / 2}\left[\alpha_{\mathrm{ds}}(\xi)-\beta_{\mathrm{ds}}(\xi) / b_{\mathrm{w}}\right] \mathrm{d} \xi\right) .
$$

Further, $\hat{S}(z)$ is assumed to be a quasihomogeneous random function of $z$, such that $\left[\operatorname{Var} \hat{S}\left(z^{\prime}\right) \operatorname{Var} \hat{S}\left(z^{\prime \prime}\right)\right]^{1 / 2} K\left(z^{\prime}, z^{\prime \prime}\right) \quad \approx \quad \operatorname{Var} \hat{S}\left[\left(z^{\prime}+\right.\right.$ $\left.\left.z^{\prime \prime}\right) / 2\right] K\left(z^{\prime}-z^{\prime \prime}\right)=\operatorname{Var} \hat{S}(\zeta) K(\eta)$ (e.g. [34]), whose correlation radius $\rho_{\mathrm{c}}$ [for $|\eta|>\rho_{\mathrm{c}} K(\eta)$ is practically equal to zero] is much smaller than $z-z_{0}$ and the variation scale of $\operatorname{Var} \hat{S}(\zeta)$. Under this assumption Eq. (26) can be written in the form

$$
\begin{aligned}
& \operatorname{Var} \widehat{J_{1}}(z)=\int_{-\left(z-z_{0}\right)}^{z-z_{0}} \mathrm{~d} \eta K(\eta) \int_{z_{0}}^{z} \mathrm{~d} \zeta \operatorname{Var} \hat{S}(\zeta) \\
& \quad \times \exp \left(2 \int_{z_{0}}^{\zeta}\left[\alpha_{\mathrm{ds}}(\xi)-\beta_{\mathrm{ds}}(\xi) / b_{\mathrm{w}}\right] \mathrm{d} \xi\right) \\
& =\rho_{\mathrm{c}} \int_{z_{0}}^{z} \mathrm{~d} \zeta \frac{N_{T}\left(E_{\mathrm{bc}}, \zeta, \Delta z\right)}{W^{2}(\zeta)} \\
& \quad \times \exp \left(2 \int_{z_{0}}^{\zeta}\left[\alpha_{\mathrm{ds}}(\xi)-\beta_{\mathrm{ds}}(\xi) / b_{\mathrm{w}}\right] \mathrm{d} \xi\right) \\
& \approx \Delta z \int_{z_{0}}^{z} \mathrm{~d} \zeta \frac{N_{T}\left(E_{\mathrm{bc}}, \zeta, \Delta z\right)}{W^{2}(\zeta)} \\
& \quad \times \exp \left(2 \int_{z_{0}}^{\zeta}\left[\alpha_{\mathrm{ds}}(\xi)-\beta_{\mathrm{ds}}(\xi) / b_{\mathrm{w}}\right] \mathrm{d} \xi\right)
\end{aligned}
$$

where $\rho_{\mathrm{c}}=\int_{-\left(z-z_{0}\right)}^{z-z_{0}} \mathrm{~d} \eta K(\eta) \approx \int_{-\infty}^{\infty} \mathrm{d} \eta K(\eta)$ is an expression of the correlation radius $\rho_{\mathrm{c}}$ that is of the order of $\Delta z$, and $\operatorname{Var} \hat{S}(\zeta)=N_{T}\left(E_{\mathrm{bc}}, \zeta, \Delta z\right) /\left[W^{2}(\zeta)\right]$ according to Eq. (20a). Next, the following expression of $\Delta z$ may be written:

$$
\Delta z=\frac{\Delta z N_{T J}(z)}{N_{T J}(z)}=N_{T J}^{-1}(z) \int_{z_{0}}^{z} N_{T}\left(E_{\mathrm{bc}}, z^{\prime}, \Delta z\right) \mathrm{d} z^{\prime},
$$

where $N_{T J}(z)=\sum_{i=0}^{\left(z-z_{0}\right) / \Delta z} N_{T}\left(E_{\mathrm{bc}}, z_{i}, \Delta z\right)$ and the fact is used that practically $\Delta z N_{T J}(z)=$ $\int_{z_{0}}^{z} N_{T}\left(E_{\mathrm{bc}}, z^{\prime}, \Delta z\right) \mathrm{d} z^{\prime}$. Taking into account in Eq. (27) the expression of Eq. (28), the inequality of Hölder for integrals [35], and Eq. (2), it follows that

$$
\begin{aligned}
& \operatorname{Var} \widehat{J}_{1}(z) \approx N_{T J}^{-1}(z) \int_{z_{0}}^{z} \mathrm{~d} \xi N_{T}\left(E_{\mathrm{bc}}, \xi, \Delta z\right) \\
& \times \int_{z_{0}}^{z} \mathrm{~d} \zeta N_{T}\left(E_{\mathrm{bc}}, \zeta, \Delta z\right) /\left[W^{2}(\zeta)\right] \\
& \quad \times \exp \left(2 \int_{z_{0}}^{\zeta}\left[\alpha_{\mathrm{ds}}(\xi)-\beta_{\mathrm{ds}}(\xi) / b_{\mathrm{w}}\right] \mathrm{d} \xi\right) \\
& \geq N_{T J}^{-1}(z)\left[\int _ { z _ { 0 } } ^ { z } \mathrm { d } \zeta S ( \zeta ) \operatorname { e x p } \left(\int _ { z _ { 0 } } ^ { \zeta } \mathrm { d } \xi \left[\alpha_{\mathrm{ds}}(\xi)\right.\right.\right. \\
& \left.\left.\left.-\beta_{\mathrm{ds}}(\xi) / b_{\mathrm{w}}\right]\right)\right]^{2}
\end{aligned}
$$




$$
\begin{aligned}
& =N_{T J}^{-1}(z) b_{\mathrm{w}}^{2}\left[1-\exp \left(-\int_{z_{0}}^{z} \mathrm{~d} \xi\left[\alpha_{\mathrm{w}}(\xi)\right.\right.\right. \\
& \left.\left.\left.+\beta_{\mathrm{ds}}(\xi) / b_{\mathrm{w}}\right]\right)\right]^{2} .
\end{aligned}
$$

After replacing in Eq. (21) the estimates and expressions (23), (24), and (29) of $S^{-2}(z) \operatorname{Var} \hat{S}(z), b_{\mathrm{w}}-J_{1}(z)$, and $\operatorname{Var} \widehat{J_{1}}(z)$, the relation $(8)$ is obtained.

The estimate (9) of the corresponding relative bias error $B_{\mathrm{r} \alpha 1}(z)$ is obtained in a similar way. In a quite analogous way as above the estimates (10) and (11) are obtained of $\delta_{\mathrm{r} \alpha 2}(z)$ and the corresponding relative bias error $B_{\mathrm{r} \alpha 2}(z)$.

\section{References}

[1] J.A. Stokes, K.R. Alvar, R.L. Corey, D.G. Costello, J. John, S. Kocimski, N.A. Lurie, D.D. Thayer, A.P. Trippe, J.C. Young, Nucl. Instrum. Methods Phys. Res. 193, 261 (1982).

[2] G. Harding, J. Kosanetzky, Nucl. Instrum. Methods Phys. Res. 280, 517 (1989).

[3] N.V. Arendtsz, E.M.A. Hussein, IEEE Trans. Nucl. Sci. 42, 2155 (1995).

[4] N.V. Arendtsz, E.M.A. Hussein, IEEE Trans. Nucl. Sci. 42, 2166 (1995).

[5] P. Zhu, P. Duvauchelle, G. Peix, D. Babot, Meas. Sci. Technol. 7, 281 (1996).

[6] S.J. Norton, J. Appl. Phys. 76, 2007 (1994).

[7] R.S. Thoe, Rev. Sci. Instrum. 67, 89 (1996).

[8] B.L. Evans, J.B. Martin, L.W. Burggrat, M.C. Roggemann, IEEE Trans. Nucl. Sci. 45, 950 (1998).

[9] B.L. Evans, J.B. Martin, L.W. Burggrat, M.C. Roggemann, T.N. Hangartner, Nucl. Instrum. Methods Phys. Res. 480, 797 (2002).

[10] G. Herman, Image Reconstruction from Projections. The Fundamentals of Computerized Tomography, Academic Press, New York 1980.

[11] F. Natterer, The Mathematics of Computerized Tomography, Wiley, Chichester 1986.

[12] B. Gustafsson, Phys. Scr. T 61, 38 (1996).

[13] L.L. Gurdev, D.V. Stoyanov, T.N. Dreischuh, Ch.N. Protochristov, O.I. Vankov, IEEE Trans. Nucl. Sci. 54, 262 (2007).

[14] L.L. Gurdev, T.N. Dreischuh, D.V. Stoyanov, Ch.N. Protochristov, in: Nuclear Methods for Non-Nuclear Applications, Ed. Ch. Stoyanov, Heron Press, Sofia 2007, p. 333.

[15] T.N. Dreischuh, L.L. Gurdev, D.V. Stoyanov, Ch.N. Protochristov, O.I. Vankov, AIP Conf. Proc. 889, 778 (2007).

[16] T.N. Dreischuh, L.L. Gurdev, D.V. Stoyanov, Ch.N. Protochristov, O.I. Vankov, Proc. SPIE 6604, 660420 (2007).
[17] G. Hertz, Lehrbuch der Kernphysik I (Experimentalle Verfahren), B.G. Teubner Verlagsgesellshaft, Leipzig 1966.

[18] P.P. Kane, Radiat. Phys. Chem. 50, 31 (1997).

[19] M. Schumacher, Phys. Rev. 182, 7 (1969).

[20] V.E. Radko, Instrum. Exp. Tech. 34, 929 (1991).

[21] J. Gerl, F. Ameil, I. Kojouharov, A. Surowiec, Nucl. Instrum. Methods Phys. Res. A 525, 328 (2004).

[22] M.J. Berger, J.H. Hubbell, S.M. Seltzer, J. Chang, J.S. Coursey, R. Sukumar, D.S. Zucker, XCOM: Photon Cross Section Database (version 1.3), [Online] Available: http://physics.nist.gov/xcom . National Institute of Standards and Technology, Gaithersburg, MD 2005.

[23] J.H. Hubbell, S.M. Seltzer, Tables of X-ray Mass Attenuation Coefficients and Mass Energy-Absorption Coefficients (version 1.4), [Online] Available: http://physics.nist.gov/xaamdi . National Institute of Standards and Technology, Gaithersburg, MD 2004.

[24] J.R. Tickner, in: Proc. 2nd RCM for the CRP Application of Nuclear Techniques to Humanitarian Demining, IAEA, St. Petersburg 2001, p. 68.

[25] K. Saito, P. Jakob, Radiat. Prot. Dosimetry 58, 29 (1995).

[26] K.F. Eckerman, J.C. Ryman, External Exposure to Radionuclides in Air, Water, and Soil, Federal Guidance Report No. 12, U.S. Environmental Protection Agency, Washington, D.C. 1993.

[27] L. Wielopolski, Z. Song, I. Orion, A.L. Hanson, G. Hendrey, Appl. Radiat. Isot. 62, 97 (2005).

[28] C.E. Ordonez, Al. Bolozdynya, W. Chang, IEEE Nucl. Sci. Conf. Record 2, 1361 (1997).

[29] V.A. Bushuev, R.N. Kuz'min, Sov. Phys. Usp. 20, 406 (1977).

[30] R. Pani, P. Bennati, M. Betti, M.N. Cinti, R. Pellegrini, M. Mattioli, V. Orsolini, F. Cencelli, D. Navarria, G. Bollini, F. Moschini, F. Garibaldi, de Notaristefani, Nucl. Instrum. Methods Phys. Res. A 567, 294 (2006).

[31] W.W. Moses, Nucl. Instrum. Methods Phys. Res. A 487, 123 (2002).

[32] S.E. Derenzo, M.J. Weber, M.K. Klintenberg, Nucl. Instrum. Methods Phys. Res. A 486, 214 (2002).

[33] W.K. Warburton, P.M. Grudberg, Nucl. Instrum. Methods Phys. Res. A 568, 350 (2006).

[34] S.M. Rytov, Yu.A. Kravtzov, V.I. Tatarski, Introduction to Statistical Radiophysics, Vol. 2, Nauka, Moscow 1978.

[35] V.A. Ilin, V.A. Sadovnichi, Bl.H. Sendov, Mathematical Analysis, Part I, Nauka i Izkustvo, Sofia 1979. 